\title{
NONSINGULAR PATH FIELDS ON COMPACT TOPOLOGICAL MANIFOLDS
}

\author{
ROBERT F. BROWN ${ }^{1}$ AND EDWARD FADELL ${ }^{2}$
}

1. Introduction. If $X$ is a topological space, a path field on $X$ is a map $s: X \rightarrow X^{I}$ such that $s(x)$ is either a path beginning at $x$ and never hitting its initial point again or $s(x)$ is the constant path at $x$. The path field $s$ is nonsingular if $s(x)$ is never constant. This concept generalizes the notion of vector field on a differentiable manifold. In [4] it was shown that a compact, connected, orientable, topological manifold (without boundary) admits a nonsingular path field if, and only if, its Euler characteristic is zero. Our objective here is to prove this theorem without the assumption of orientability and, in addition, for compact manifolds with boundary.

2. The Lefschetz formula. Let $X$ denote an ANR (compact metric) and $f: X \rightarrow X$ a given map. Then, F. Browder [2] and also Leray and Deleanu [12], [6] have developed a local index theory which assigns to every open set $O \subset X$, such that $f$ has no fixed points on its boundary $\partial O$, an integer $i(f, O)$ called the index of $f$ on $O$. This index function has very nice properties listed explicitly in $[2$, p. 256]. Incidentally, the theory only requires $f$ to be defined on $\bar{O}$, the closure of $O$, i.e., $f: \bar{O} \rightarrow X$.

Suppose we add the assumption that $f$ has an isolated fixed point $x_{0}$ and $X$ is locally Euclidean at $x_{0}$, i.e., there is an open set $U$, containing $x_{0}$, homeomorphic to Euclidean $n$-space $R^{n}$. Then, there is an index $I_{f}\left(x_{0}\right)$ for $f$ at $x_{0}$ defined in terms of local degree ([13], [15]).

Proposition (2.1). If $O$ is any open set in $X$ containing $x_{0}$, then for $O$ sufficiently small,

$$
i(f, O)=(-1)^{n} I_{f}\left(x_{0}\right) .
$$

Proof. Let $h: R^{n} \rightarrow U$ denote a homeomorphism such that $h(0)$ $=x_{0}$. Let $C$ denote the closed unit ball in $R^{n}, C^{0}$ its interior, and $V=h\left(C^{0}\right) \subset U$. We may assume that $V$ contains no fixed points of $f$ other than $x_{0}$. We will show that if $O$ is any open set containing $x_{0}$ such that $\bar{O} \cup f(\bar{O}) \subset V$, then $i(f, O)=(-1)^{n} I_{f}\left(x_{0}\right)$. Let $O_{1}=h^{-1}(O)$ $C C^{0} \cdot h^{-1} f: \bar{O} \rightarrow C$ has the property that $h\left(h^{-1} f\right)=f$ has no fixed points on $\partial O$. Therefore, by the Commutative Property of $i[2]$

\footnotetext{
Presented to the Society, August 27, 1964; received by the editors April 16, 1964.

1 Supported, in part, by the Air Force Office of Scientific Research.

2 Supported by the National Science Foundation under Grant NSF G-23849 and the Wisconsin Alumni Research Foundation.
} 


$$
i\left(\left(h^{-1} f\right) h, O_{1}\right)=i\left(h\left(h^{-1} f\right), O\right)=i(f, 0) .
$$

Let $g=h^{-1} f h: \bar{O}_{1} \rightarrow C$. If $G: C \rightarrow C$ is any extension of $g$, then $i(f, O)$ $=i\left(G, O_{1}\right)$. Since $C$ is a polyhedron, $i\left(G, O_{1}\right)$ is the local index restricted to the category of polyhedra and continuous maps. But, O'Neill [13] has shown that a local index function satisfying appropriate conditions (satisfied by the Browder-Leray-Deleanu index) is unique on this category of polyhedra. His uniqueness proof shows, in fact, that $i\left(G, O_{1}\right)=(-1)^{n} I_{G}(0)$. But by definition, $I_{f}\left(x_{0}\right)=I_{G}(0)$. Therefore, $i(f, O)=(-1)^{n} I_{f}\left(x_{0}\right)$.

Proposition (2.2) (The Lefschetz formula). Let $X$ denote an $A N R$ (compact metric), $f: X \rightarrow X$ a given map with a finite number of fixed points, $x_{1}, \cdots, x_{r}$. If each $x_{j}, 1 \leqq j \leqq r$, possesses an open neighborhood $U_{j}$ homeomorphic to $R^{n(j)}$, then

$$
\Lambda(f)=\sum_{j=1}^{n}(-1)^{n(j)} I_{f}\left(x_{j}\right),
$$

where $\Lambda(f)$ is the Lefschetz number of $f$.

Proof. Choose sufficiently small, mutually disjoint open sets $O_{j}$ such that $x_{j} \in O_{j}$ and $i\left(f, O_{j}\right)=(-1)^{n(j)} I_{f}\left(x_{j}\right)$. Then, the whole space $X$ is an open set and $X-U_{j=1}^{r} O_{j}$ has no fixed points of $f$. Therefore, by the Additivity Property [2] of the index $i$,

$$
i(f, X)=\sum_{j=1}^{r} i\left(f, O_{j}\right) \text {. }
$$

But the Normalization Property [2] tells us that $i(f, X)=\Lambda(f)$, the Lefschetz number of $f$. Thus,

$$
\Lambda(f)=i(f, X)=\sum_{j=1}^{r} i\left(f, O_{j}\right)=\sum_{j=1}^{r}(-1)^{n(j)} I_{f}\left(x_{j}\right) .
$$

Corollary (2.3). Let $M$ denote a compact topological n-manifold with a finite number of fixed points $x_{1}, \cdots, x_{r}$. Then

$$
\Lambda(f)=(-1)^{n} \sum_{j=1}^{r} I_{f}\left(x_{j}\right)
$$

If $M$ is a compact topological manifold with boundary $B$, the result is still valid provided the fixed points $x_{1}, \cdots, x_{r}$, do not belong to $B$.

REMARK (2.4). Proposition (2.2) generalizes the form of the Lefschetz formula proved in [5], as well as Theorem 3 in [15]. 
3. A basic theorem. We recall first [7] that if $\mathcal{F}=(E, p, B)$, $\mathcal{F}_{0}=\left(E_{0}, p_{0}, B\right)$ are regular fiber spaces, in the sense of Hurewicz [10] over the same base $B$, then $\left(F, \mathcal{F}_{0}\right)=\left(E, E_{0}, p, B\right)$ is a fibered pair provided $E_{0} \subset E, p_{0}=p \mid E_{0}$ and $\mathcal{F}$ admits a lifting function $\lambda$ such that $\lambda$ lifts paths in $B$ into $E_{0}$ if the preassigned initial point is in $E_{0}$. $\left(F, F_{0}\right)=\left(p^{-1}(b), p_{0}^{-1}(b)\right), b \in B$, is the fiber of $\left(F, \mathcal{F}_{0}\right)$. A generalized $n$-plane bundle $\left(n\right.$-gpb) [7] is a fibered pair $\left(\mathcal{F}, \mathcal{F}_{0}\right)$ with fiber $\left(F, F_{0}\right)$ such that

(i) there exists a cross section $\sigma: B \rightarrow E$ such that $E_{0}=E-\sigma(B)$,

(ii) $\left(F, F_{0}\right) \sim\left(R^{n}, R^{n}-0\right)$.

If $s: B \rightarrow E$ is a cross section, $b \in B$ is called a singular point of $s$ if $s(b) \notin E_{0}$. Our objective here is the following.

Theorem (3.1). Let $\left(F, \mathcal{F}_{0}\right)=\left(E, E_{0}, p, M\right)$ denote an $n$-gpb, $n \geqq 2$, with fiber $\left(F, F_{0}\right)$ and base $M$, where $M$ is a compact connected topological n-manifold with boundary $B$. Suppose $s_{0}: B \rightarrow E_{0}$ is a given partial cross section (without singularities). Then, there is a cross section $s: M$ $\rightarrow E$ with at most one singularity, which extends $s_{0}$.

Proof. The simpler case $B=\varnothing$ is given in [4] and we therefore assume $B \neq \varnothing$.

I. First we observe that by [3] the boundary $B$ is "collared," i.e., there is an imbedding $h: B \times[0,1] \rightarrow M$ such that $h(b, 0)=b, b \in M$. Let $A=h(B \times[0,1]), A^{0}=h(B \times[0,1))$. Choose an open cover $\chi$ (all coverings are considered finite) of $M$ so fine that if $U \in \chi$ and $U \cap B$ $\neq \varnothing$, then the star of $U$ (rel $\chi)$ is contained in $A^{0}$. Since $M$ is an ANR (compact metric), and hence $L C^{*}[11]$, there are open covers $\alpha_{0}>\beta_{0}>\chi\left(\alpha_{0}\right.$ refines $\beta_{0}, \beta_{0}$ refines $\left.\chi\right)$ such that if $\alpha>\alpha_{0}$ and $N_{\alpha}$ is the geometric nerve of $\alpha$, there are maps

$$
N_{\alpha} \underset{p_{\alpha}}{\stackrel{q_{\alpha}}{\rightleftarrows}} M
$$

with the following properties.

(a) $q_{\alpha} p_{\alpha} \sim{ }_{\chi} 1$.

(b) If $x \in M, p_{\alpha}(x) \in\left|V_{i_{0}}, \cdots, V_{i_{k}}\right|$, where $V_{i_{0}}, \cdots, V_{i_{k}}$ are all the open sets in $\alpha$ containing $x$, and $\left|V_{i_{0}}, \cdots, V_{i_{k}}\right|$ is the $k$-simplex determined by the "vertices" $V_{i_{j}}$.

(c) If $V \in N_{\alpha}$, is a "vertex," $q_{\alpha}(V) \in V$ and for any simplex $\tau$ of $N_{\alpha}, q_{\alpha}(\bar{\tau})$ belongs to some member of $\beta_{0}$.

Since $M$ has dimension $n$, we may choose $\alpha$ so that $K=N_{\alpha}$ is an $n$-complex, $\alpha>\alpha_{0}$. Let $L$ denote the following subcomplex of $K . L$ is the union of all closed stars of vertices $V \in \alpha$ such that $V \cap B \neq \varnothing$. 
Let $f=q_{\alpha}, g=p_{\alpha}$, where $q_{\alpha}, p_{\alpha}$ are chosen to satisfy (a), (b), and (c). Then

$$
\begin{gathered}
K \underset{g}{\stackrel{f}{\rightleftarrows}} M, \\
f g \sim_{\chi} 1, \\
f(L) \subset A^{0}, \\
g(B) \subset L .
\end{gathered}
$$

II. We now show that the given cross section $s_{0}: B \rightarrow E_{0}$ can be extended to $s_{1}: A \rightarrow E_{0}$. This is done by applying the following simple lemma.

Lemma (3.2). If $\mathcal{F}=\left(E_{0}, p_{0}, A\right)$ is a regular fiber space and $B \subset A$ is a strong deformation retract of $A$, then a partial cross section $s_{0}: B \rightarrow E_{0}$ is always extendable to a cross section $s_{1}: A \rightarrow E_{0}$.

III. The map $f: K \rightarrow M$ induces an $n-\mathrm{gpb}\left(\digamma^{*}, \digamma_{0}^{*}\right)=\left(E^{*}, E_{0}^{*}, p^{*}, K\right)$ over $K$ with fiber $\left(F, F_{0}\right)$ using the given fibered pair $\left(\mathcal{F}, \mathcal{F}_{0}\right)$ over $M$. Since $f(L) \subset A$, the partial cross section $s_{1}: A \rightarrow E_{0}$ induces a cross section $s_{1}^{*}: L \rightarrow E_{0}^{*}$. Since $\pi_{i}\left(F_{0}\right)=0$ for $i<n-1, s_{1}^{*}$ has an extension $s^{*}: K^{[n-1]} \cup L \rightarrow E_{0}^{*}$. If $\sigma^{n}$ is an $n$-simplex of $K-L$, a simple argument extends $s^{*}$ to $\sigma^{n}$ where $s^{*}$ takes on values in $E_{0}^{*}$ except at the barycenter of $\sigma^{n}$. Thus we obtain a cross section $s^{*}: K \rightarrow E$ such that $s^{*}$ has only a finite number of singularities $y_{1}, \cdots, y_{r}$, each the barycenter of a $n$-simplex of $K-L$.

IV. As is usual with induced fibrations, there is an associated commutative diagram

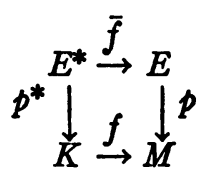

where $\bar{f}\left(E_{0}^{*}\right) \subset E_{0}$. Let us investigate the map $\phi=\bar{f} s^{*} g$. First, we remark that $\phi$ is not a cross section, in fact, $p \phi=p \bar{f} s^{*} g=f p^{*} s^{*} g=f g \sim_{\chi} 1$. Let $H: M \times I \rightarrow M$ denote a homotopy with the following properties. If $\tilde{H}: M \rightarrow M^{I}$ is the corresponding map into the space of paths then,

(i) for $x \in M, \tilde{H}(x)$ begins at $f g(x)$ and ends at $x$,

(ii) for $x \in B, \tilde{H}(x) \in A^{I}$.

Now, if $\lambda$ is a regular lifting function for $\left(F, \mathcal{F}_{0}\right)$, define a cross section $\psi: M \rightarrow E$ by

$$
\psi(x)=\lambda[\phi(x), \tilde{H}(x)](1), \quad x \in M .
$$


Thus, $\phi$ can be "pulled over" to a cross section $\psi$ with the property that if $\phi(x) \in E_{0}$, then $\psi(x) \in E_{0}, x \in M$. Let $\psi_{0}=\psi \mid B: B \rightarrow E_{0}$. We wish to show that $s_{0}$ and $\psi_{0}$ are homotopic as cross sections into $E_{0}$. If $x \in B, \phi(x)=\bar{f} s^{*} g(x)=\bar{f} s_{1}^{*} g(x)=s_{1} f g(x)$. Thus, $s_{1} \tilde{H}(x)$ is a path in $E_{0}$ beginning at $\phi(x)$ and ending at $s_{1}(x)=s_{0}(x) . \lambda[\phi(x), \tilde{H}(x)]$ is a path in $E_{0}$ beginning at $\phi(x)$ and ending at $\psi_{0}(x)$. Both of these paths lie over $\tilde{H}(x)$. Applying Proposition 1 of [8], we obtain $s_{0} \sim \psi_{0}: B \rightarrow E_{0}$ as cross sections. We note, at this point, the singularities of $\psi$. Let $Q_{j}=g^{-1}\left(y_{j}\right)$, where $y_{1}, \cdots, y_{r}$ are the singularities of $s^{*}$ (III). The sets $Q_{j}$ are closed, mutually disjoint, and none of them intersect the boundary $B$. Furthermore, the initial cover $\chi$ may be chosen so that each set $Q_{j}$ is a compact subset of a Euclidean neighborhood which does not intersect the boundary. Thus, if $Q=\cup Q_{j}$, we see that the singularities of $\psi$ lie in $Q$.

V. Now, using the techniques in the proof of Theorem 1.10 [4], there is a cross section $\zeta: M \rightarrow E$, with a finite number of singularities, which agrees with $\psi$ on the boundary $B$. The proof of Lemma 1.9 [4] will yield a cross section $s^{\prime}: M \rightarrow E$ with one singularity such that $s^{\prime}$ agrees with $\zeta$ (and hence with $\psi$ ) on the boundary $B$. Thus, if $s_{0}^{\prime}$ $=s^{\prime} \mid B, s_{0}^{\prime} \sim s_{0}$ as cross sections into $E_{0}$.

VI. To complete the proof we proceed as follows. Let $x_{0} \in M-B$ denote the singular point for $s^{\prime}$ and choose a small concentric $n$-cell neighborhood $\bar{V} \supset V \supset \bar{U} \supset U$ such that $x_{0} \in U$. Then $(M-U, B)$ is an ANR pair. Applying the fiber homotopy extension theorem (FHET) in [1]. There is a homotopy

$$
G:(M-U) \times I \rightarrow E_{0}
$$

such that $G_{0}=s^{\prime} \mid M-U, G_{t}$ is a cross section over $M-U$ for each $t, 0 \leqq t \leqq 1$, and $G_{1} \mid B=s_{0}$. Now, $(M, M-U)$ is again an ANR pair and $G_{0}$ has $s^{\prime}$ as an extension to $M$. Again applying the FHET, this time in the fiber space $(E, p, B), G$ has an extension to a homotopy

$$
G^{\prime}: M \times I \rightarrow E
$$

such that $G_{1}^{\prime}=s^{\prime \prime}$ is a cross section over $M$ which extends $s_{0}$ and has all its singularities in $U$. $s^{\prime \prime}$ can be redefined in $U$, using the techniques already referred to, to produce a cross section $s$ which has $x_{0} \in U$ as its only singular point and which extends $s_{0}$.

4. The main result. Let $X$ denote a topological space and

$$
T_{0}(X)=\left\{\omega \in X^{I}: \omega(t)=\omega(0) \text { if, and only if, } t=0,0 \leqq t \leqq 1\right\} \text {. }
$$

Let $p_{0}: T_{0}(X) \rightarrow X$ be given by $p_{0}(\omega)=\omega(0)$. Let $T(X)$ denote $T_{0}(X)$ plus all the constant paths on $X$ and $p: T(X) \rightarrow X$ be given by $p(\omega)$ 
$=\omega(0)$. A path field on $X$ is a cross section for $p$, i.e., a map $s: X$ $\rightarrow T(X)$ such that $p s=1$. A singularity for $s$ is a point $x$ such that $s(x)$ is the constant path at $x$. A nonsingular path field is a path field without singularities.

If $X=M$, a topological $n$-manifold (with empty boundary), then $\left(\Im, J_{0}\right)=\left(T(M), T_{0}(M), p, M\right)$ is an $n$-gpb [7], called the tangent fiber space of $M$.

Suppose that $M$ is a compact topological $n$-manifold with boundary $B$. Let $h: B \times I \rightarrow M$ denote an imbedding which puts a "collar" on $B$ and $h(x, 0)=x, x \in B$. Let $A=h(B \times I), A^{0}=h(B \times[0,1)), A_{1}$ $=h(B \times\{1\})$. Then, $h$ may be chosen so that if $M^{\prime}=M-A^{0}, B^{\prime}=A_{1}$, then $\left(M^{\prime}, B^{\prime}\right)$ is a homeomorphic copy of $(M, B)$. Let $\left(J, J_{0}\right)=\left(T, T_{0}\right.$, $p, M-B)$ denote the tangent fiber space of $M-B$ and $\left(J^{\prime}, J_{0}^{\prime}\right)$ $=\left(T^{\prime}, T_{0}^{\prime}, p^{\prime}, M^{\prime}\right)$ the restriction of $\left(J^{\prime}, \Im_{0}\right)$ to $M^{\prime}$.

LemMa (4.1). Suppose $s_{0}: B^{\prime} \rightarrow T_{0}\left(M^{\prime}\right)$ is a given nonsingular path field (in $M^{\prime}$ ) on $B^{\prime}$. Since $T_{0}\left(M^{\prime}\right) \subset T_{0}^{\prime}, s_{0}$ can be considered as a partial cross section over $B^{\prime}$ in the fibered pair $\left(J^{\prime}, J_{0}^{\prime}\right)$. Then, $s_{0}$ admits an extension to a path field $s: M^{\prime} \rightarrow T_{0}\left(M^{\prime}\right)$ if, and only if, $s_{0}: B^{\prime} \rightarrow T_{0}^{\prime}$ admits an extension to a cross section $s^{\prime}: M^{\prime} \rightarrow T_{0}^{\prime}$.

Proof. The necessity is trivial. Suppose, therefore, that $s_{0}: B^{\prime} \rightarrow T_{0}^{\prime}$ admits an extension, $s^{\prime}: M^{\prime} \rightarrow T_{0}^{\prime}$. $s^{\prime}$ would serve for $s$ except for the fact that some of the paths might wander outside of $M^{\prime}$. Such paths, however, start in $M^{\prime}-B^{\prime}$. Let $\rho: M-B \rightarrow M^{\prime}$ denote a retraction such that $\rho$ maps points outside of $M^{\prime}$ into $B^{\prime}$. Then $\rho$ induces $\rho^{\prime}:(M-B)^{I} \rightarrow M^{\prime I}$ and $s=\rho^{\prime} s^{\prime}: M^{\prime} \rightarrow T_{0}\left(M^{\prime}\right)$, is the required extension of $s_{0}$.

ThEOREM (4.2). Let $M$ denote a compact connected n-manifold ( $n \geqq 2)$ with boundary $B$ and $s_{0}: B \rightarrow T_{0}(M)$ a given nonsingular path field (in $M$ ) on $B$. Then, $s_{0}$ can be extended to a nonsingular path field $s: M \rightarrow T_{0}(M)$ if, and only if, the Euler characteristic $\chi(M)=0$.

Proof. The proof for an empty boundary $B$ is simpler and is implicit in the argument for the case $B \neq \varnothing$. We, therefore, assume $B \neq \varnothing$.

The necessity is immediate since here we get a fixed point free map of $M$ into itself homotopic to the identity.

To prove the sufficiency, identify $(M, B)$ with $\left(M^{\prime}, B^{\prime}\right)$ of the previous lemma. Using the notation in the previous lemma $s_{0}$ can be considered as a partial cross section $s_{0}: B^{\prime} \rightarrow T_{0}^{\prime}$, and it suffices to extend $s_{0}$ to $s: M^{\prime} \rightarrow T_{0}^{\prime}$. Now, $\left(J^{\prime}, J_{0}^{\prime}\right)$ is an $n$-gpb and hence by our basic Theorem (3.1), there is a cross section $s^{\prime}: M^{\prime} \rightarrow T^{\prime}$ with at most 
one singularity (in $M^{\prime}-B^{\prime}$ ) and $s^{\prime} \mid B^{\prime}=s_{0}$. We may assume (see proof of (4.1)) that $s^{\prime}\left(M^{\prime}\right) \subset T\left(M^{\prime}\right)$. $s^{\prime}$ gives rise to a map $f: M^{\prime} \rightarrow M^{\prime}$ by setting $f(x)=s^{\prime}(x)(1), x \in M^{\prime}$, and $f \sim 1 . f$ has a single fixed point $x_{0}$ and by $(1.3) \Lambda_{f}=(-1)^{n} I_{f}\left(x_{0}\right)$. But $\chi(M)=\Lambda_{f}$ and hence the assumption that $\chi(M)=0$ implies $I_{f}\left(x_{0}\right)=0$. Now, using an argument identical with that in $[4,2.3], I_{f}\left(x_{0}\right)=0$ is a sufficient condition for redefining $s^{\prime}$ in a neighborhood of $x_{0}$ to produce an extension $s: M^{\prime}$ $\rightarrow T_{0}^{\prime}$ of $s_{0}$. Thus, our theorem follows.

Since a compact topological $n$-manifold $M$ with boundary $B$ always admits a nonsingular path field $s_{0}: B \rightarrow T_{0}(M)$ on its boundary we have the following.

ThEOREM (4.3). A compact connected topological $n$-manifold $M$, with or without boundary (not necessarily orientable), admits a nonsingular path field if, and only if, $\chi(M)=0$. In the case of a manifold with boundary, the nonsingular path field may be preassigned on its boundary.

If $M$ is a compact topological manifold with boundary $B$, a path field $s: M \rightarrow T(M)$ is called regular if $x \in B$ implies $s(x)$ is a path in $B$.

TheOREM (4.4). A compact connected topological $n$-manifold $M$ with boundary components $B_{1}, \cdots, B_{r}$ admits a regular nonsingular path field if, and only if, $\chi(M)=0$ and $\chi\left(B_{j}\right)=0,1 \leqq j \leqq r$.

TheOREM (4.5). A compact connected topological $n$-manifold $M$ with connected boundary $B$ admits a regular nonsingular path field if, and only if, $\chi(M)=0$.

Proof. We prove only the sufficiency and it suffices to show $\chi(B)$ $=0$. If $n$ is even, $n-1$ is odd and $\chi(B)=0$. Otherwise, if $n$ is odd, let $2 M$ denote the double of $M$. Then

$$
\chi(2 M)=2 \chi(M)-\chi(B) .
$$

Therefore, since $\chi(2 M)=0$ and $\chi(M)=0$, we have $\chi(B)=0$.

The following theorem bears on a question of M. Hirsch [9]. It is an immediate corollary of (4.3).

Corollary (4.6). A compact connected topological $n$-manifold $M$, with or without boundary (not necessarily orientable) admits a map $f: M \rightarrow M$ which is fixed point free and homotopic to the identity if, and only if, $\chi(M)=0$.

REMARK (4.7). An example of Y. H. Clifton, namely $S^{2} \bigvee S^{1} \bigvee S^{1}$ shows that (4.6) is false in general for polyhedra. However, for a large class of polyhedra (zweidimensional zusammenhängede) Wecken [14] has proved (4.6). Our result (4.3) is much stronger than (4.6). 
REMARK (4.8). The analogue of (4.3) for compact differentiable $n$-manifolds and nonsingular vector fields is well known, at least in the case of no boundary. The result for differentiable manifolds with boundary is also classical (H. Hopf, Math. Ann. 96 (1927), 225$250)$. Both of these results are obtainable as corollaries of our results for topological manifolds by showing that the tangent bundles in question are fiber homotopy equivalent to appropriate tangent fiber spaces. The no-boundary case is given in [4], [7] and the case of a differentiable manifold $M$ with boundary $B$ is handled as follows. First "shrink" $(M, B)$ to another copy $\left(M^{\prime}, B^{\prime}\right)$ via a tubular neighborhood of $B$. Then, the tangent btundle of $M$ may be identified with the tangent bundle of $M$ restricted to $M^{\prime}$. This restricted tangent bundle is easily seen to be fiber homotopy equivalent to the tangent fiber space of $M-B$, restricted to $M^{\prime}$. Lemma (4.1) completes the necessary paraphernalia.

\section{BIBLIOGRAPHY}

1. G. Allaud and E. Fadell, $A$ fiber homotopy extension theorem, Trans. Amer. Math. Soc. 104 (1962), 239-251.

2. F. E. Browder, On the fixed point index for continuous mappings of locally connected spaces, Summa Brasil. Math. 4 (1960), 253-293.

3. M. Brown, Locally fat embeddings of topological manifolds, Topology of 3 . Manifolds, M. Fort, Ed., Prentice-Hall, Englewood Cliffs, N. J., 1962; pp. 83-91.

4. R. Brown, Path fields on manifolds, Trans. Amer. Math. Soc. 118 (1965), 180191.

5. - On the Lefschets fixed point formula, Amer. J. Math. 87 (1965), 1-10.

6. A. Deleanu, Theorie des points fixes sur les retracts de voisinage des espace convexoide, Bull. Soc. Math. France 87 (1959), 235-243.

7. E. Fadell, Generalized normal bundles for locally flat imbeddings, Trans. Amer. Math Soc. 114 (1965), 488-513.

8. - On fiber spaces, Trans. Amer. Math. Soc. 90 (1959), 1-14.

9. M. Hirsch, Problem 18, Combinatorial and differentiable structures, Problems in Differential and Algebraic Topology (mimeographed), Summer Topology Institute, 1962, Seattle, Wash.

10. W. Hurewicz, On the concept of fiber space, Proc. Nat. Acad. Sci. U.S.A. 41 (1955), 956-961.

11. S. Lefschetz, Topics in topology, Annals of Mathematical Studies, No. 10, Princeton Univ. Press, Princeton, N. J., 1942.

12. J. Leray, Theorie des points fixes. Indice total et nombre de Lefschetz, Bull. Soc. Math. France 87 (1959), 221-233.

13. B. O'Neill, Essential sets and fixed points, Amer. J. Math. 75 (1953), 497-509.

14. F. Wecken, Fixpunktklassen. III, Math. Ann. 118 (1941-1943), 544-577.

15. J. Weier, Fixpunkttheorie in topologischen Mannigfaltigkeiten, Math. Z. 59 (1953), 171-190.

University of California, Los Angeles, INSTITUTE FOR ADVANCED STUdY AND UNIVERSITY OF WISCONSIN 